\title{
Epitaxial Growth of Atomically Smooth Aluminum on Si and Its Intrinsic Optical Properties
}

Fei Cheng,,$^{\dagger, \perp}$ Ping-Hsiang Su,,$^{\dagger, \perp}$ Junho Choi, ${ }^{\dagger}$ Shangjr Gwo, ${ }^{\dagger}$ Xiaoqin $L i,{ }^{\dagger}$ and

Chih-Kang Shih ${ }^{*}, \dagger$

${ }^{\dagger}$ Department of Physics, University of Texas at Austin, Texas 78712 United States

${ }^{\ddagger}$ Department of Physics, National Tsing-Hua University, Hsinchu 30013, Taiwan

*E-mail: $\underline{\text { shih@physics.utexas.edu }}$ 


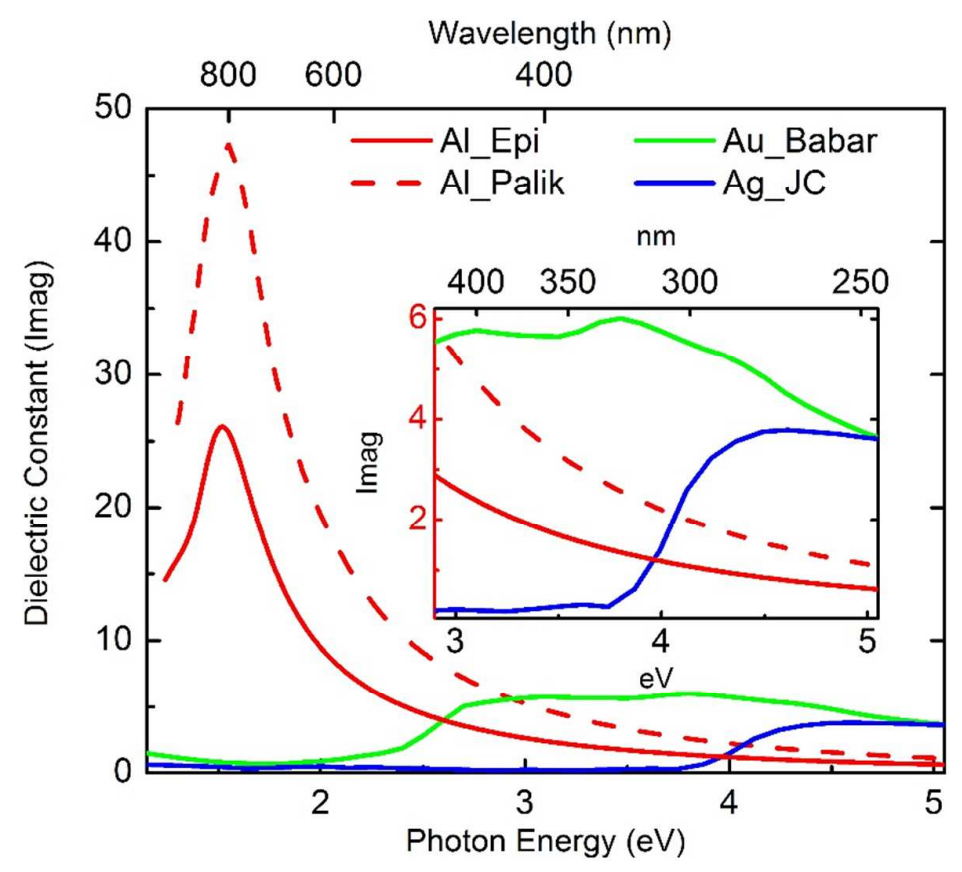

Figure S1. Comparison of optical constants (imaginary part) between gold, silver, and aluminum. The inset shows an enlarged view at the UV energy range. For gold and silver, their respective interband transitions starting from $2.3 \mathrm{eV}(\sim 540 \mathrm{~nm})$ and $4 \mathrm{eV}$ $(\sim 310 \mathrm{~nm})$ lead to significant intrinsic ohmic losses in the ultraviolet regime, where aluminum exhibits suppressed losses and thus superior plasmonic properties. 

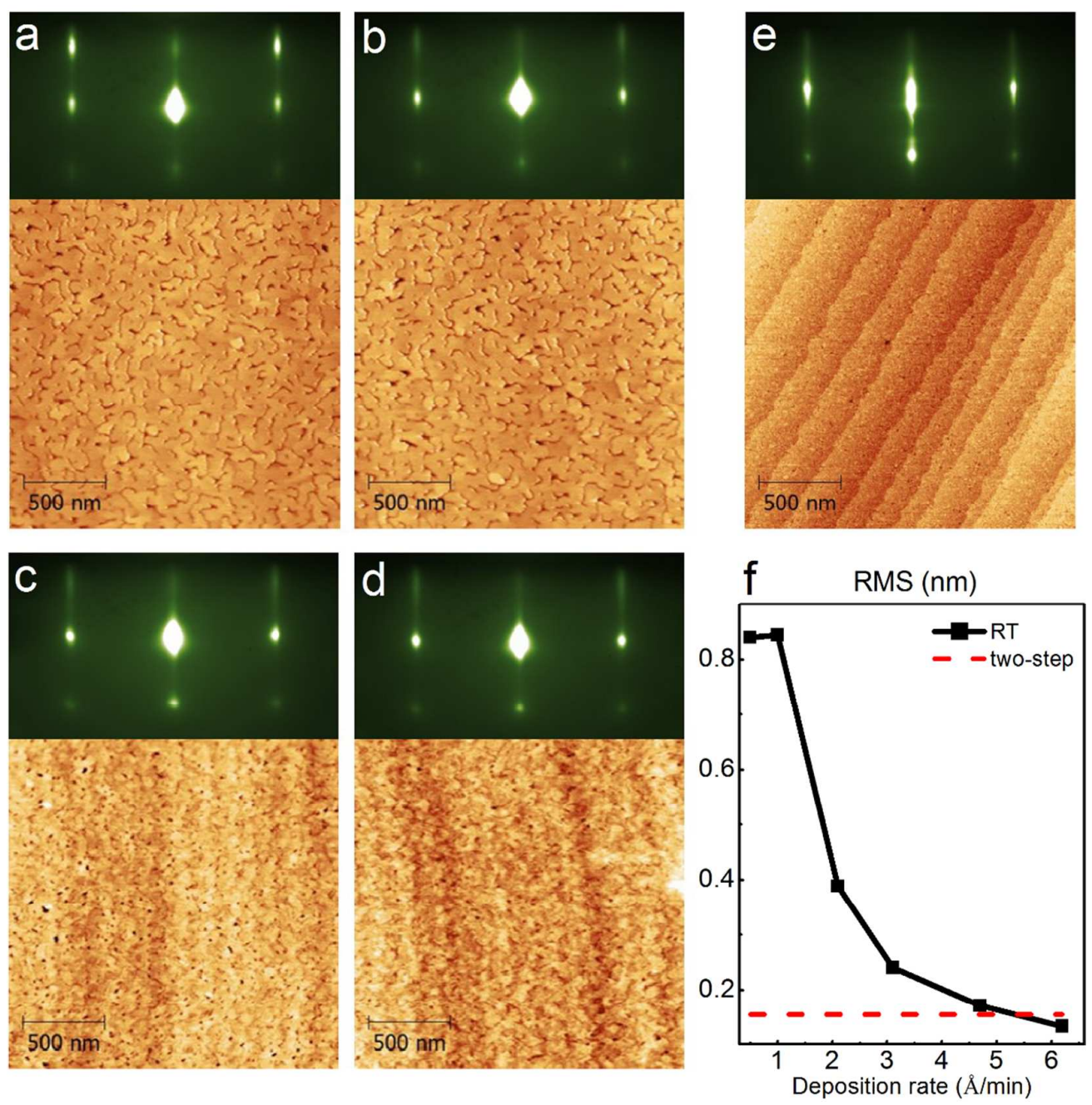

Figure S2. RHEED patterns and AFM scans of Al films deposited at RT with different

Knudsen cell temperatures and deposition rates: (a) $1120^{\circ} \mathrm{C}, 0.5 \AA / \mathrm{min}$; (b) $1150^{\circ} \mathrm{C}$,

$1.0 \AA / \mathrm{min}$; (c) $1220^{\circ} \mathrm{C}, 4.7 \AA / \mathrm{min}$; (d) $1240^{\circ} \mathrm{C}, 6.2 \AA / \mathrm{min}$. (e) $\mathrm{Al}$ films $(\mathrm{RMS}=0.155 \mathrm{~nm}$ )

prepared by two-step method for comparison. Scale bar: $500 \mathrm{~nm}$. We note that

fissure-like structures are observed for films grown with lower deposition rates (2a and

2b) and tiny pinholes are observed for higher deposition rates (2c and 2d). (g) RMS

values according to the AFM scans at respective deposition rate. Cases of $1175^{\circ} \mathrm{C}$, 
$2.1 \AA / \mathrm{min}$ and $1200^{\circ} \mathrm{C}, 3.1 \AA / \mathrm{min}$ are included. The red dashed line represents the RMS value of the two-step method.
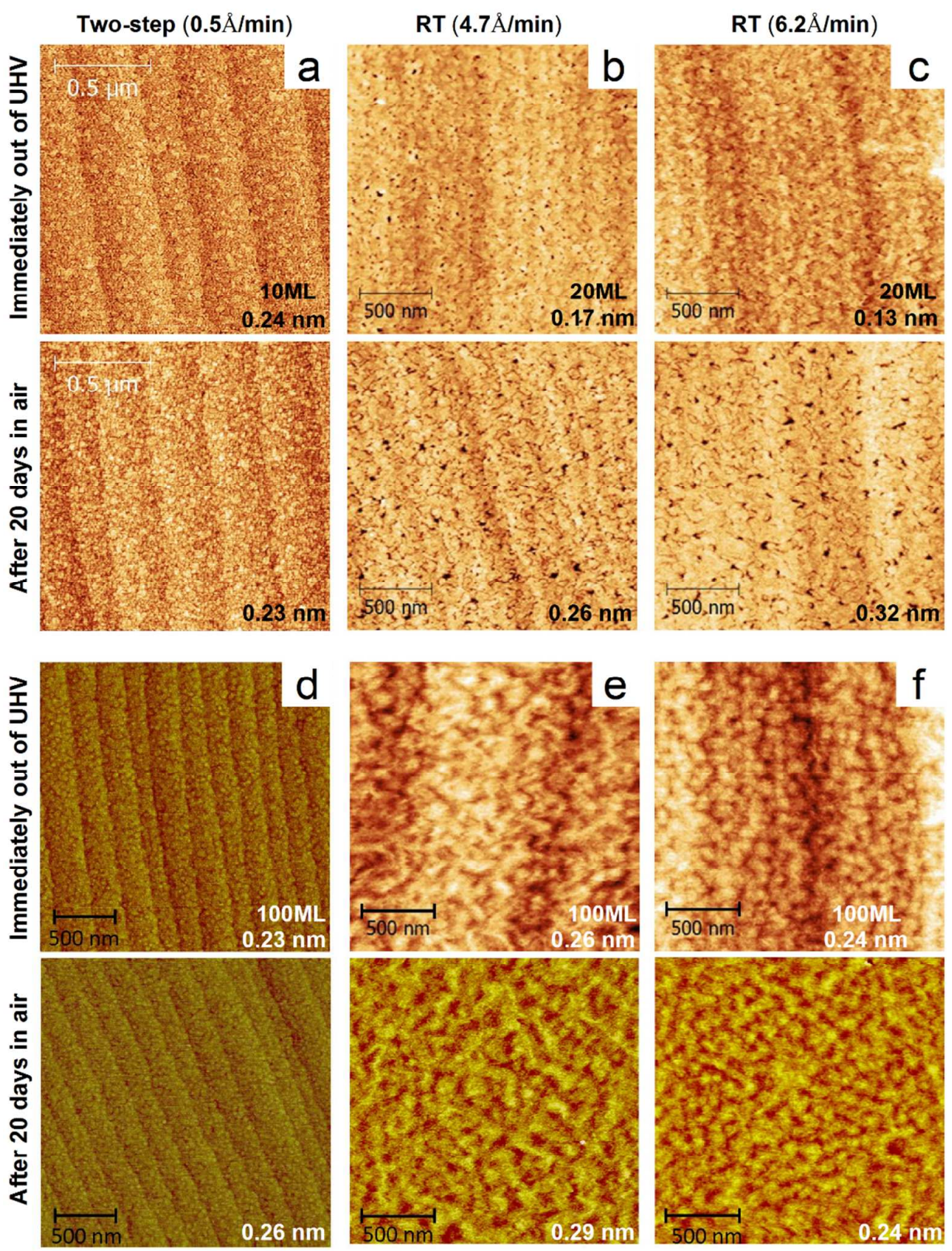
Figure S3. (a) AFM images of a 10ML Al film (two-step method, deposition rate $0.5 \AA / \mathrm{min}$ ) taken immediately after the film was taken out the UHV chamber (top) and that taken 20 days after the film was taken out of chamber (bottom). (b,c) AFM images of two $20 \mathrm{ML}$ Al films grown by the RT method with a deposition rate of $4.7 \AA / \mathrm{min}$ (b) and a rate of $6.2 \AA / \mathrm{min}$ (c). (d-f) AFM images of a 100ML Al film deposited using the two-step method $(0.5 \AA / \mathrm{min}, \mathrm{d})$ and two $100 \mathrm{ML} \mathrm{Al}$ films grown by the RT method with a deposition rate of $4.7 \AA / \mathrm{min}$ (e) and a rate of $6.2 \AA / \mathrm{min}$ (f). The number in the right bottom corner of each figure indicates the thickness and RMS roughness of each film.
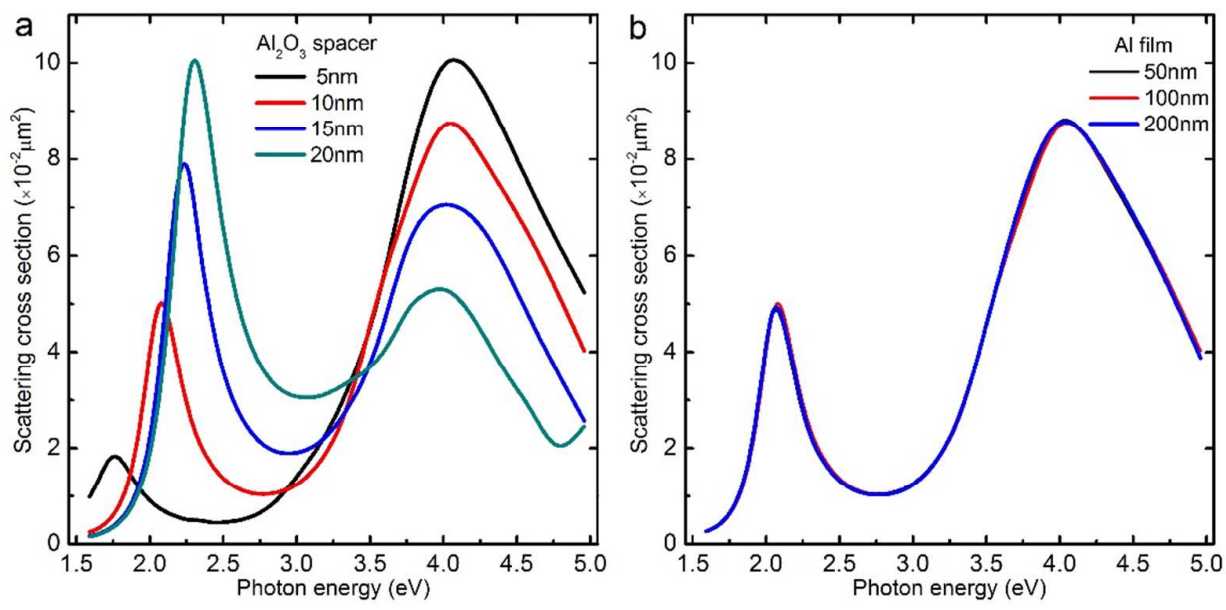

Figure S4. (a) The scattering cross sections for an Al nanocube (edge size $d=75 \mathrm{~nm}$ ) sitting on an Al film $(t=100 \mathrm{~nm})$. A thin oxide layer is sandwiched between the nanocube and the film with varying thicknesses from $5 \mathrm{~nm}$ to $20 \mathrm{~nm}$. (b) The scattering cross sections for an $\mathrm{Al}$ nanocube $(d=75 \mathrm{~nm})$ sitting $\mathrm{Al}$ films with varying thicknesses from $50 \mathrm{~nm}$ to $200 \mathrm{~nm}$ (the oxide layer thickness is kept at $10 \mathrm{~nm}$ ). Measured optical constants from the epitaxial Al film are adopted in calculations of this figure. 
According to Figure S4a, the relative intensity of the quadrupole mode increases while that of the dipole mode decreases as the oxide spacer decreases from $20 \mathrm{~nm}$ to 5 $\mathrm{nm}$. Meanwhile, an obvious redshift of the dipolar mode is observed $(2.31 \mathrm{eV}$ to 1.75 $\mathrm{eV}$ ) and a tiny blueshift is found for the quadrupolar mode (3.98 eV to $4.07 \mathrm{eV})$. We attribute the observation to the fact that a smaller distance (thinner oxide layer) between the nanocube and the film is expected to lead to an enhanced interaction between them, resulting in a larger spectral separation between the two modes and a stronger intensity of the quadrupole mode. According to Figure S4b, we found that the thickness of underlying $\mathrm{Al}$ film barely effects the scattering cross sections of the $\mathrm{Al}$ nanocube. As a result, the thickness of oxide layer plays a more important role in the spectral response of the Al nanocube-film hybrids.

\section{Table S1. The complete optical constants look-up table (1.24-5.5 eV) for epitaxially grown, single-crystalline Al film}

We provide below the tabulated optical constants extracted from the 100ML, epitaxial Al film grown using the two-step method. Here we did not provide an explicit expression of analytical fit due to its complicated form. The Drude model is most responsible for loss in the near infrared (IR) range while the Lorentz and the Tauc-Lorentz models mainly contribute to the optical transitions from near IR to UV range. Alternative models with simpler analytical expressions can be used but with larger fitting errors. 


\begin{tabular}{|c|c|c|}
\hline Photon Energy (eV) & $\varepsilon_{1}$ & $\varepsilon_{2}$ \\
\hline 1.24 & -58.288 & 14.544 \\
\hline 1.25 & -57.318 & 14.822 \\
\hline 1.26 & -56.357 & 15.091 \\
\hline 1.27 & -55.412 & 15.35 \\
\hline 1.28 & -54.477 & 15.603 \\
\hline 1.29 & -53.555 & 15.85 \\
\hline 1.3 & -52.642 & 16.097 \\
\hline 1.31 & -51.726 & 16.351 \\
\hline 1.32 & -50.815 & 16.62 \\
\hline 1.33 & -49.911 & 16.91 \\
\hline 1.34 & -49.011 & 17.232 \\
\hline 1.35 & -48.12 & 17.597 \\
\hline 1.36 & -47.254 & 18.009 \\
\hline 1.37 & -46.419 & 18.476 \\
\hline 1.38 & -45.634 & 18.998 \\
\hline 1.39 & -44.906 & 19.578 \\
\hline 1.4 & -44.258 & 20.208 \\
\hline 1.41 & -43.701 & 20.881 \\
\hline 1.42 & -43.25 & 21.581 \\
\hline 1.43 & -42.914 & 22.291 \\
\hline 1.44 & -42.696 & 22.993 \\
\hline 1.45 & -42.598 & 23.663 \\
\hline 1.46 & -42.615 & 24.277 \\
\hline
\end{tabular}




\begin{tabular}{|c|c|c|}
\hline 1.47 & -42.733 & 24.822 \\
\hline 1.48 & -42.939 & 25.281 \\
\hline 1.49 & -43.216 & 25.641 \\
\hline 1.5 & -43.542 & 25.895 \\
\hline 1.51 & -43.896 & 26.04 \\
\hline 1.52 & -44.266 & 26.083 \\
\hline 1.53 & -44.627 & 26.024 \\
\hline 1.54 & -44.972 & 25.875 \\
\hline 1.55 & -45.287 & 25.645 \\
\hline 1.56 & -45.565 & 25.343 \\
\hline 1.57 & -45.797 & 24.984 \\
\hline 1.58 & -45.983 & 24.575 \\
\hline 1.59 & -46.121 & 24.128 \\
\hline 1.6 & -46.21 & 23.655 \\
\hline 1.61 & -46.252 & 23.155 \\
\hline 1.62 & -46.249 & 22.644 \\
\hline 1.63 & -46.204 & 22.124 \\
\hline 1.64 & -46.12 & 21.603 \\
\hline 1.65 & -45.998 & 21.079 \\
\hline 1.66 & -45.845 & 20.56 \\
\hline 1.67 & -45.662 & 20.05 \\
\hline 1.68 & -45.453 & 19.55 \\
\hline 1.69 & -45.219 & 19.054 \\
\hline 1.7 & -44.966 & 18.577 \\
\hline 1.71 & -44.692 & 18.107 \\
\hline
\end{tabular}




\begin{tabular}{|c|c|c|}
\hline 1.72 & -44.405 & 17.654 \\
\hline 1.73 & -44.102 & 17.211 \\
\hline 1.74 & -43.787 & 16.783 \\
\hline 1.75 & -43.462 & 16.369 \\
\hline 1.76 & -43.127 & 15.965 \\
\hline 1.77 & -42.787 & 15.578 \\
\hline 1.78 & -42.441 & 15.204 \\
\hline 1.79 & -42.089 & 14.841 \\
\hline 1.8 & -41.732 & 14.49 \\
\hline 1.81 & -41.374 & 14.151 \\
\hline 1.82 & -41.014 & 13.825 \\
\hline 1.83 & -40.652 & 13.509 \\
\hline 1.84 & -40.288 & 13.204 \\
\hline 1.85 & -39.928 & 12.911 \\
\hline 1.86 & -39.564 & 12.626 \\
\hline 1.87 & -39.204 & 12.351 \\
\hline 1.88 & -38.843 & 12.085 \\
\hline 1.89 & -38.483 & 11.827 \\
\hline 1.9 & -38.126 & 11.579 \\
\hline 1.91 & -37.771 & 11.339 \\
\hline 1.92 & -37.419 & 11.106 \\
\hline 1.93 & -37.07 & 10.882 \\
\hline 1.94 & -36.722 & 10.663 \\
\hline 1.95 & -36.378 & 10.452 \\
\hline 1.96 & -36.037 & 10.248 \\
\hline
\end{tabular}




\begin{tabular}{|c|c|c|}
\hline 1.97 & -35.698 & 10.049 \\
\hline 1.98 & -35.363 & 9.8569 \\
\hline 1.99 & -35.03 & 9.6702 \\
\hline 2 & -34.702 & 9.4895 \\
\hline 2.01 & -34.376 & 9.3136 \\
\hline 2.02 & -34.055 & 9.1435 \\
\hline 2.03 & -33.736 & 8.9775 \\
\hline 2.04 & -33.42 & 8.8167 \\
\hline 2.05 & -33.111 & 8.6613 \\
\hline 2.06 & -32.802 & 8.5089 \\
\hline 2.07 & -32.498 & 8.3619 \\
\hline 2.08 & -32.197 & 8.2184 \\
\hline 2.09 & -31.899 & 8.0782 \\
\hline 2.1 & -31.605 & 7.9424 \\
\hline 2.11 & -31.315 & 7.8102 \\
\hline 2.12 & -31.027 & 7.6812 \\
\hline 2.13 & -30.744 & 7.5557 \\
\hline 2.14 & -30.464 & 7.4335 \\
\hline 2.15 & -30.186 & 7.3141 \\
\hline 2.16 & -29.913 & 7.1983 \\
\hline 2.17 & -29.642 & 7.0847 \\
\hline 2.18 & -29.376 & 6.9746 \\
\hline 2.19 & -29.111 & 6.8666 \\
\hline 2.2 & -28.851 & 6.7615 \\
\hline 2.21 & -28.593 & 6.6587 \\
\hline
\end{tabular}




\begin{tabular}{|c|c|c|}
\hline 2.22 & -28.338 & 6.5583 \\
\hline 2.23 & -28.086 & 6.4602 \\
\hline 2.24 & -27.838 & 6.3646 \\
\hline 2.25 & -27.593 & 6.2715 \\
\hline 2.26 & -27.351 & 6.1801 \\
\hline 2.27 & -27.111 & 6.0909 \\
\hline 2.28 & -26.876 & 6.0042 \\
\hline 2.29 & -26.642 & 5.9187 \\
\hline 2.3 & -26.412 & 5.8356 \\
\hline 2.31 & -26.183 & 5.7536 \\
\hline 2.32 & -25.957 & 5.6737 \\
\hline 2.33 & -25.735 & 5.5956 \\
\hline 2.34 & -25.515 & 5.5191 \\
\hline 2.35 & -25.298 & 5.4445 \\
\hline 2.36 & -25.083 & 5.3708 \\
\hline 2.37 & -24.87 & 5.2991 \\
\hline 2.38 & -24.661 & 5.2287 \\
\hline 2.39 & -24.453 & 5.1597 \\
\hline 2.4 & -24.249 & 5.0924 \\
\hline 2.41 & -24.047 & 5.0264 \\
\hline 2.42 & -23.847 & 4.9615 \\
\hline 2.43 & -23.649 & 4.8977 \\
\hline 2.44 & -23.454 & 4.8356 \\
\hline 2.45 & -23.262 & 4.7745 \\
\hline 2.46 & -23.07 & 4.7143 \\
\hline
\end{tabular}




\begin{tabular}{|c|c|c|}
\hline 2.47 & -22.882 & 4.6557 \\
\hline 2.48 & -22.695 & 4.5976 \\
\hline 2.49 & -22.511 & 4.5412 \\
\hline 2.5 & -22.329 & 4.4856 \\
\hline 2.51 & -22.149 & 4.4309 \\
\hline 2.52 & -21.971 & 4.3775 \\
\hline 2.53 & -21.795 & 4.3249 \\
\hline 2.54 & -21.622 & 4.2735 \\
\hline 2.55 & -21.449 & 4.2226 \\
\hline 2.56 & -21.28 & 4.1728 \\
\hline 2.57 & -21.111 & 4.1237 \\
\hline 2.58 & -20.945 & 4.0758 \\
\hline 2.59 & -20.781 & 4.0286 \\
\hline 2.6 & -20.618 & 3.982 \\
\hline 2.61 & -20.457 & 3.9364 \\
\hline 2.62 & -20.298 & 3.8915 \\
\hline 2.63 & -20.141 & 3.8474 \\
\hline 2.64 & -19.986 & 3.8043 \\
\hline 2.65 & -19.832 & 3.7616 \\
\hline 2.66 & -19.679 & 3.7195 \\
\hline 2.67 & -19.53 & 3.6785 \\
\hline 2.68 & -19.381 & 3.6379 \\
\hline 2.69 & -19.233 & 3.598 \\
\hline 2.7 & -19.088 & 3.5588 \\
\hline 2.71 & -18.943 & 3.52 \\
\hline
\end{tabular}




\begin{tabular}{|c|c|c|}
\hline 2.72 & -18.801 & 3.4821 \\
\hline 2.73 & -18.66 & 3.4447 \\
\hline 2.74 & -18.521 & 3.408 \\
\hline 2.75 & -18.383 & 3.3718 \\
\hline 2.76 & -18.246 & 3.3361 \\
\hline 2.77 & -18.111 & 3.3011 \\
\hline 2.78 & -17.977 & 3.2665 \\
\hline 2.79 & -17.845 & 3.2324 \\
\hline 2.8 & -17.714 & 3.1989 \\
\hline 2.81 & -17.584 & 3.1658 \\
\hline 2.82 & -17.456 & 3.1334 \\
\hline 2.83 & -17.329 & 3.1014 \\
\hline 2.84 & -17.203 & 3.0697 \\
\hline 2.85 & -17.079 & 3.0386 \\
\hline 2.86 & -16.956 & 3.008 \\
\hline 2.87 & -16.834 & 2.9778 \\
\hline 2.88 & -16.713 & 2.9481 \\
\hline 2.89 & -16.594 & 2.9188 \\
\hline 2.9 & -16.476 & 2.89 \\
\hline 2.91 & -16.359 & 2.8614 \\
\hline 2.92 & -16.244 & 2.8335 \\
\hline 2.93 & -16.129 & 2.8058 \\
\hline 2.94 & -16.015 & 2.7786 \\
\hline 2.95 & -15.902 & 2.7515 \\
\hline 2.96 & -15.791 & 2.725 \\
\hline
\end{tabular}




\begin{tabular}{|c|c|c|}
\hline 2.97 & -15.681 & 2.699 \\
\hline 2.98 & -15.572 & 2.6732 \\
\hline 2.99 & -15.464 & 2.6477 \\
\hline 3 & -15.356 & 2.6225 \\
\hline 3.01 & -15.25 & 2.5978 \\
\hline 3.02 & -15.145 & 2.5733 \\
\hline 3.03 & -15.041 & 2.5493 \\
\hline 3.04 & -14.939 & 2.5256 \\
\hline 3.05 & -14.837 & 2.5021 \\
\hline 3.06 & -14.736 & 2.479 \\
\hline 3.07 & -14.636 & 2.4563 \\
\hline 3.08 & -14.537 & 2.4338 \\
\hline 3.09 & -14.439 & 2.4115 \\
\hline 3.1 & -14.341 & 2.3895 \\
\hline 3.11 & -14.245 & 2.3679 \\
\hline 3.12 & -14.15 & 2.3466 \\
\hline 3.13 & -14.056 & 2.3256 \\
\hline 3.14 & -13.962 & 2.3046 \\
\hline 3.15 & -13.869 & 2.2841 \\
\hline 3.16 & -13.777 & 2.2638 \\
\hline 3.17 & -13.686 & 2.2438 \\
\hline 3.18 & -13.596 & 2.224 \\
\hline 3.19 & -13.507 & 2.2045 \\
\hline 3.2 & -13.419 & 2.1852 \\
\hline 3.21 & -13.331 & 2.1662 \\
\hline
\end{tabular}




\begin{tabular}{|c|c|c|}
\hline 3.22 & -13.244 & 2.1473 \\
\hline 3.23 & -13.158 & 2.1287 \\
\hline 3.24 & -13.072 & 2.1103 \\
\hline 3.25 & -12.987 & 2.0922 \\
\hline 3.26 & -12.904 & 2.0743 \\
\hline 3.27 & -12.82 & 2.0566 \\
\hline 3.28 & -12.738 & 2.0391 \\
\hline 3.29 & -12.656 & 2.0218 \\
\hline 3.3 & -12.576 & 2.0048 \\
\hline 3.31 & -12.495 & 1.9879 \\
\hline 3.32 & -12.416 & 1.9712 \\
\hline 3.33 & -12.337 & 1.9547 \\
\hline 3.34 & -12.259 & 1.9385 \\
\hline 3.35 & -12.182 & 1.9224 \\
\hline 3.36 & -12.105 & 1.9065 \\
\hline 3.37 & -12.029 & 1.8908 \\
\hline 3.38 & -11.953 & 1.8753 \\
\hline 3.39 & -11.878 & 1.8599 \\
\hline 3.4 & -11.804 & 1.8448 \\
\hline 3.41 & -11.731 & 1.8298 \\
\hline 3.42 & -11.658 & 1.815 \\
\hline 3.43 & -11.586 & 1.8003 \\
\hline 3.44 & -11.514 & 1.7858 \\
\hline 3.45 & -11.443 & 1.7715 \\
\hline 3.46 & -11.373 & 1.7574 \\
\hline
\end{tabular}




\begin{tabular}{|c|c|c|}
\hline 3.47 & -11.303 & 1.7433 \\
\hline 3.48 & -11.234 & 1.7295 \\
\hline 3.49 & -11.165 & 1.7158 \\
\hline 3.5 & -11.097 & 1.7022 \\
\hline 3.51 & -11.03 & 1.6888 \\
\hline 3.52 & -10.963 & 1.6756 \\
\hline 3.53 & -10.897 & 1.6625 \\
\hline 3.54 & -10.831 & 1.6496 \\
\hline 3.55 & -10.765 & 1.6367 \\
\hline 3.56 & -10.7 & 1.624 \\
\hline 3.57 & -10.636 & 1.6115 \\
\hline 3.58 & -10.572 & 1.5991 \\
\hline 3.59 & -10.509 & 1.5868 \\
\hline 3.6 & -10.447 & 1.5747 \\
\hline 3.61 & -10.385 & 1.5627 \\
\hline 3.62 & -10.323 & 1.5508 \\
\hline 3.63 & -10.262 & 1.5391 \\
\hline 3.64 & -10.201 & 1.5274 \\
\hline 3.65 & -10.141 & 1.5159 \\
\hline 3.66 & -10.081 & 1.5045 \\
\hline 3.67 & -10.022 & 1.4933 \\
\hline 3.68 & -9.9631 & 1.4821 \\
\hline 3.69 & -9.9048 & 1.471 \\
\hline 3.7 & -9.847 & 1.4601 \\
\hline 3.71 & -9.7897 & 1.4493 \\
\hline
\end{tabular}




\begin{tabular}{|c|c|c|}
\hline 3.72 & -9.7329 & 1.4386 \\
\hline 3.73 & -9.6766 & 1.4281 \\
\hline 3.74 & -9.6205 & 1.4176 \\
\hline 3.75 & -9.5648 & 1.4072 \\
\hline 3.76 & -9.5098 & 1.397 \\
\hline 3.77 & -9.455 & 1.3868 \\
\hline 3.78 & -9.4005 & 1.3767 \\
\hline 3.79 & -9.3465 & 1.3667 \\
\hline 3.8 & -9.2932 & 1.3569 \\
\hline 3.81 & -9.2401 & 1.3471 \\
\hline 3.82 & -9.1874 & 1.3374 \\
\hline 3.83 & -9.1351 & 1.3278 \\
\hline 3.84 & -9.0832 & 1.3184 \\
\hline 3.85 & -9.0317 & 1.309 \\
\hline 3.86 & -8.9806 & 1.2997 \\
\hline 3.87 & -8.93 & 1.2905 \\
\hline 3.88 & -8.8796 & 1.2814 \\
\hline 3.89 & -8.8298 & 1.2724 \\
\hline 3.9 & -8.7802 & 1.2634 \\
\hline 3.91 & -8.7311 & 1.2546 \\
\hline 3.92 & -8.6823 & 1.2459 \\
\hline 3.93 & -8.6339 & 1.2372 \\
\hline 3.94 & -8.5859 & 1.2286 \\
\hline 3.95 & -8.5382 & 1.2201 \\
\hline 3.96 & -8.4908 & 1.2117 \\
\hline
\end{tabular}




\begin{tabular}{|c|c|c|}
\hline 3.97 & -8.4439 & 1.2033 \\
\hline 3.98 & -8.3972 & 1.1951 \\
\hline 3.99 & -8.3509 & 1.1869 \\
\hline 4 & -8.3048 & 1.1788 \\
\hline 4.01 & -8.2592 & 1.1707 \\
\hline 4.02 & -8.2138 & 1.1628 \\
\hline 4.03 & -8.1688 & 1.1549 \\
\hline 4.04 & -8.124 & 1.1471 \\
\hline 4.05 & -8.0797 & 1.1393 \\
\hline 4.06 & -8.0358 & 1.1317 \\
\hline 4.07 & -7.9922 & 1.1241 \\
\hline 4.08 & -7.9489 & 1.1166 \\
\hline 4.09 & -7.9057 & 1.1091 \\
\hline 4.1 & -7.8628 & 1.1017 \\
\hline 4.11 & -7.8202 & 1.0944 \\
\hline 4.12 & -7.7782 & 1.0871 \\
\hline 4.13 & -7.7364 & 1.08 \\
\hline 4.14 & -7.6948 & 1.0728 \\
\hline 4.15 & -7.6533 & 1.0657 \\
\hline 4.16 & -7.6124 & 1.0588 \\
\hline 4.17 & -7.5717 & 1.0518 \\
\hline 4.18 & -7.5313 & 1.045 \\
\hline 4.19 & -7.4913 & 1.0382 \\
\hline 4.2 & -7.4515 & 1.0315 \\
\hline 4.21 & -7.4118 & 1.0248 \\
\hline
\end{tabular}




\begin{tabular}{|c|c|c|}
\hline 4.22 & -7.3723 & 1.0181 \\
\hline 4.23 & -7.3335 & 1.0116 \\
\hline 4.24 & -7.2948 & 1.0051 \\
\hline 4.25 & -7.2563 & 0.99861 \\
\hline 4.26 & -7.2179 & 0.99219 \\
\hline 4.27 & -7.1799 & 0.98585 \\
\hline 4.28 & -7.1423 & 0.97959 \\
\hline 4.29 & -7.1049 & 0.97336 \\
\hline 4.3 & -7.0678 & 0.96721 \\
\hline 4.31 & -7.0308 & 0.96108 \\
\hline 4.32 & -6.9941 & 0.95501 \\
\hline 4.33 & -6.9576 & 0.94898 \\
\hline 4.34 & -6.9213 & 0.943 \\
\hline 4.35 & -6.8855 & 0.93712 \\
\hline 4.36 & -6.8499 & 0.93126 \\
\hline 4.37 & -6.8144 & 0.92547 \\
\hline 4.38 & -6.7792 & 0.9197 \\
\hline 4.39 & -6.7441 & 0.91398 \\
\hline 4.4 & -6.7093 & 0.9083 \\
\hline 4.41 & -6.6746 & 0.90265 \\
\hline 4.42 & -6.6404 & 0.89711 \\
\hline 4.43 & -6.6063 & 0.89159 \\
\hline 4.44 & -6.5725 & 0.88612 \\
\hline 4.45 & -6.539 & 0.88071 \\
\hline 4.46 & -6.5056 & 0.87532 \\
\hline
\end{tabular}




\begin{tabular}{|c|c|c|}
\hline 4.47 & -6.4724 & 0.86999 \\
\hline 4.48 & -6.4393 & 0.86469 \\
\hline 4.49 & -6.4065 & 0.85943 \\
\hline 4.5 & -6.3741 & 0.85424 \\
\hline 4.51 & -6.3418 & 0.84908 \\
\hline 4.52 & -6.3097 & 0.84397 \\
\hline 4.53 & -6.2777 & 0.83888 \\
\hline 4.54 & -6.2459 & 0.83382 \\
\hline 4.55 & -6.2145 & 0.82885 \\
\hline 4.56 & -6.1832 & 0.8239 \\
\hline 4.57 & -6.1521 & 0.81898 \\
\hline 4.58 & -6.1212 & 0.8141 \\
\hline 4.59 & -6.0904 & 0.80925 \\
\hline 4.6 & -6.0599 & 0.80446 \\
\hline 4.61 & -6.0296 & 0.7997 \\
\hline 4.62 & -5.9994 & 0.79497 \\
\hline 4.63 & -5.9695 & 0.79031 \\
\hline 4.64 & -5.9397 & 0.78566 \\
\hline 4.65 & -5.9102 & 0.78104 \\
\hline 4.66 & -5.8809 & 0.7765 \\
\hline 4.67 & -5.8517 & 0.77197 \\
\hline 4.68 & -5.8228 & 0.76748 \\
\hline 4.69 & -5.794 & 0.76303 \\
\hline 4.7 & -5.7653 & 0.75859 \\
\hline 4.71 & -5.7369 & 0.7542 \\
\hline
\end{tabular}




\begin{tabular}{|c|c|c|}
\hline 4.72 & -5.7086 & 0.74985 \\
\hline 4.73 & -5.6804 & 0.74551 \\
\hline 4.74 & -5.6525 & 0.74124 \\
\hline 4.75 & -5.6248 & 0.73699 \\
\hline 4.76 & -5.5972 & 0.73276 \\
\hline 4.77 & -5.5698 & 0.72858 \\
\hline 4.78 & -5.5425 & 0.72442 \\
\hline 4.79 & -5.5153 & 0.72028 \\
\hline 4.8 & -5.4884 & 0.7162 \\
\hline 4.81 & -5.4617 & 0.71214 \\
\hline 4.82 & -5.435 & 0.7081 \\
\hline 4.83 & -5.4087 & 0.70412 \\
\hline 4.84 & -5.3824 & 0.70016 \\
\hline 4.85 & -5.3563 & 0.69622 \\
\hline 4.86 & -5.3304 & 0.69232 \\
\hline 4.87 & -5.3045 & 0.68844 \\
\hline 4.88 & -5.2788 & 0.68457 \\
\hline 4.89 & -5.2533 & 0.68076 \\
\hline 4.9 & -5.228 & 0.67697 \\
\hline 4.91 & -5.2027 & 0.6732 \\
\hline 4.92 & -5.1778 & 0.66948 \\
\hline 4.93 & -5.153 & 0.66579 \\
\hline 4.94 & -5.1283 & 0.66212 \\
\hline 4.95 & -5.1037 & 0.65848 \\
\hline 4.96 & -5.0793 & 0.65486 \\
\hline
\end{tabular}




\begin{tabular}{|c|c|c|}
\hline 4.97 & -5.0549 & 0.65126 \\
\hline 4.98 & -5.0307 & 0.64769 \\
\hline 4.99 & -5.0067 & 0.64415 \\
\hline 5 & -4.9828 & 0.64063 \\
\hline 5.01 & -4.9591 & 0.63714 \\
\hline 5.02 & -4.9355 & 0.63368 \\
\hline 5.03 & -4.912 & 0.63024 \\
\hline 5.04 & -4.8886 & 0.62681 \\
\hline 5.05 & -4.8655 & 0.62344 \\
\hline 5.06 & -4.8425 & 0.62009 \\
\hline 5.07 & -4.8195 & 0.61674 \\
\hline 5.08 & -4.7968 & 0.61344 \\
\hline 5.09 & -4.7741 & 0.61016 \\
\hline 5.1 & -4.7516 & 0.60689 \\
\hline 5.11 & -4.7292 & 0.60365 \\
\hline 5.12 & -4.7069 & 0.60043 \\
\hline 5.13 & -4.6848 & 0.59723 \\
\hline 5.14 & -4.6627 & 0.59405 \\
\hline 5.15 & -4.6409 & 0.59092 \\
\hline 5.16 & -4.6191 & 0.58779 \\
\hline 5.17 & -4.5975 & 0.58468 \\
\hline 5.18 & -4.576 & 0.58161 \\
\hline 5.19 & -4.5546 & 0.57855 \\
\hline 5.2 & -4.5333 & 0.57551 \\
\hline 5.21 & -4.5121 & 0.57248 \\
\hline
\end{tabular}




\begin{tabular}{|c|c|c|}
\hline 5.22 & -4.4911 & 0.5695 \\
\hline 5.23 & -4.4702 & 0.56653 \\
\hline 5.24 & -4.4493 & 0.56357 \\
\hline 5.25 & -4.4287 & 0.56064 \\
\hline 5.26 & -4.4081 & 0.55774 \\
\hline 5.27 & -4.3877 & 0.55485 \\
\hline 5.28 & -4.3673 & 0.55197 \\
\hline 5.29 & -4.3471 & 0.54913 \\
\hline 5.3 & -4.327 & 0.5463 \\
\hline 5.31 & -4.3069 & 0.54347 \\
\hline 5.32 & -4.287 & 0.54069 \\
\hline 5.33 & -4.2673 & 0.53793 \\
\hline 5.34 & -4.2476 & 0.53518 \\
\hline 5.35 & -4.228 & 0.53244 \\
\hline 5.36 & -4.2086 & 0.52973 \\
\hline 5.37 & -4.1892 & 0.52703 \\
\hline 5.38 & -4.1699 & 0.52435 \\
\hline 5.39 & -4.1507 & 0.52168 \\
\hline 5.4 & -4.1318 & 0.51905 \\
\hline 5.41 & -4.1128 & 0.51643 \\
\hline 5.42 & -4.094 & 0.51383 \\
\hline 5.43 & -4.0753 & 0.51124 \\
\hline 5.44 & -4.0567 & 0.50868 \\
\hline 5.45 & -4.0381 & 0.50613 \\
\hline 5.46 & -4.0197 & 0.50359 \\
\hline
\end{tabular}




\begin{tabular}{|c|c|c|}
\hline 5.47 & -4.0013 & 0.50105 \\
\hline 5.48 & -3.983 & 0.49853 \\
\hline 5.49 & -3.9647 & 0.49602 \\
\hline 5.5 & -3.9465 & 0.49351 \\
\hline
\end{tabular}

